\title{
Toward a Universal Model for Spatially Structured Populations
}

\author{
Loïc Marrec $\oplus^{1, \dagger}$ Irene Lamberti, ${ }^{1, \hbar}$ and Anne-Florence Bitbol $\oplus^{1,2,3, *}$ \\ ${ }^{1}$ Sorbonne Université, CNRS, Institut de Biologie Paris-Seine, Laboratoire Jean Perrin (UMR 8237), F-75005 Paris, France \\ ${ }^{2}$ Institute of Bioengineering, School of Life Sciences, École Polytechnique Fédérale de Lausanne (EPFL), \\ CH-1015 Lausanne, Switzerland \\ ${ }^{3}$ SIB Swiss Institute of Bioinformatics, CH-1015 Lausanne, Switzerland
}

(Received 19 April 2021; revised 23 July 2021; accepted 6 October 2021; published 17 November 2021)

\begin{abstract}
A key question in evolution is how likely a mutant is to take over. This depends on natural selection and on stochastic fluctuations. Population spatial structure can impact mutant fixation probabilities. We introduce a model for structured populations on graphs that generalizes previous ones by making migrations independent of birth and death. We demonstrate that by tuning migration asymmetry, the star graph transitions from amplifying to suppressing natural selection. The results from our model are universal in the sense that they do not hinge on a modeling choice of microscopic dynamics or update rules. Instead, they depend on migration asymmetry, which can be experimentally tuned and measured.
\end{abstract}

DOI: 10.1103/PhysRevLett.127.218102

Introduction.-Classical models of well-mixed, homogeneous microbial populations assume that each microorganism competes with all others. However, this simplification holds in few natural situations. For instance, during an infection, microbial populations are subdivided between different organs [1,2] and hosts. Any spatial structure, e.g., that of a Petri dish, implies a stronger competition between neighbors than between distant individuals. Even well-agitated liquid suspensions feature deviations compared with idealized well-mixed populations [3].

Spatial structure can have major consequences on evolution. Remarkably, the fixation probability of a mutant can be affected, with specific structures amplifying or suppressing natural selection [4]. Studying these effects requires going beyond simple structures $[5,6]$ where migration is symmetric between demes (i.e., subpopulations), since fixation probabilities are unaffected in these cases [7-9], unless extinctions of demes occur [10]. Reference [4] introduced a seminal model for complex structures, known as evolutionary dynamics on graphs, with one individual at each node of a graph, and probabilities that their offspring replaces a neighbor along each edge of the graph. However, in such models, evolutionary outcomes can drastically depend on the details of the microscopic dynamics or "update rule," e.g., whether the individual that divides or the one that dies is chosen first, even if selection always acts at division [11-14]. This lack of universality raises

Published by the American Physical Society under the terms of the Creative Commons Attribution 4.0 International license. Further distribution of this work must maintain attribution to the author(s) and the published article's title, journal citation, and DOI. issues for applicability to real populations, where one birth does not necessarily entail one death and vice versa. Furthermore, in most microbial populations, individuals freely compete with their closest neighbors, motivating a coarse-grained description, with demes rather than individuals on graph nodes [5,6,15-18]. Current experiments with well-mixed demes at each node of a star graph [19] require theoretical predictions with realistic microscopic dynamics.

We propose a model for complex spatial population structures where migrations are independent from birth and death events. We investigate the fixation probability of mutants in the rare migration regime. We demonstrate that migration asymmetry determines whether the star graph amplifies or suppresses natural selection. We find a mapping to the model of Ref. [4] under specific constraints on migration rates.

Model.-We model a structured population as a directed graph where each node $i \in\{1, \ldots, D\}$ contains a wellmixed deme with carrying capacity $K$, and migration rates $m_{i j}$ per individual from deme $i$ to deme $j \neq i$ are specified along each edge $i j$. We then address populations including demes with different carrying capacities [20]. We consider microorganisms with two types, wild type $(W)$ and mutant $(M)$, with fitnesses and death rates denoted by $f_{a}$ and $g_{a}$, where $a=W$ or $a=M$. Here, we call fitness the maximal division rate of microorganisms, reached in exponential growth. Their division rate in deme $i$ is given by the logistic function $f_{a}\left(1-N_{i} / K\right)$, where $N_{i}$ is the number of individuals in deme $i$. We take wild-type fitness as a reference, $f_{W}=1$. We address selection on birth, and hence $g_{M}=g_{W}$, but our results can be generalized to selection on death. We focus on the regime where deme sizes $N_{i}$ fluctuate weakly around their deterministic steady-state values, without extinctions $[10,26,27]$. 
We assume that mutations are rare enough for further mutation events to be neglected while the fate of a given mutant lineage (taking over or disappearing) is determined. We consider an initial mutant placed uniformly at random, which is realistic for spontaneous mutations occurring either with a fixed rate or with a fixed probability upon division. Note that in models with one individual per node, uniform initialization is more appropriate in the first case, while placing mutants proportionally to the replacement probability of a node ("temperature initialization") is more appropriate in the second one [28]. This distinction vanishes here, as division rate does not depend on location. Under uniform initialization, the fixation probability of a neutral mutant is independent of structure for connected graphs [20]. Compared with the well-mixed population with the same total size, an amplifier of natural selection features a larger fixation probability for beneficial mutants $\left(f_{M}>f_{W}\right)$, and a smaller one for deleterious mutants $\left(f_{M}<f_{W}\right)$, while a suppressor has the opposite characteristics [29].

We focus on the rare migration regime [9], where fixation of a type ( $W$ or $M$ ) in a deme is much faster than migration timescales. Then, the state of the population can be described in a coarse-grained way by whether each deme is mutant or wild type. Its evolution is a Markov process where elementary steps are migration events, which change the state of the system if fixation ensues. In this regime, a mutant first needs to fix in the deme where it appeared, before mutants can spread to other demes. Since fixation in a homogeneous deme is well-known, we study the second stage, starting from one fully mutant deme.

Link with models with one individual per node.-A formal mapping can be made between our model and that of Ref. [4], if the same graph is considered, with a deme per node in our model and with one individual per node in Ref. [4] (see Ref. [20]). The probability $\mathcal{P}_{i \rightarrow j}$ that, upon a migration event resulting into fixation, an individual from deme $i$ takes over in deme $j$ in our model maps to the probability $P_{i \rightarrow j}^{[4]}$ that, upon a division, the offspring from node $i$ replaces the individual on node $j$ in the model of Ref. [4]:

$$
\mathcal{P}_{i \rightarrow j}=\frac{m_{i j} N_{i} \rho_{i}}{\sum_{k, l} m_{k l} N_{k} \rho_{k}} \leftrightarrow P_{i \rightarrow j}^{[4]}=\frac{w_{i j} f_{i}}{\sum_{k, l} w_{k l} f_{k}} .
$$

In this mapping, the product $N_{i} \rho_{i}$ of deme size $N_{i}$ and fixation probability $\rho_{i}$ of an organism from deme $i$ in our model plays the part of fitness $f_{i}$ of the individual on node $i$ in Ref. [4], while the migration rate $m_{i j}$ plays the part of the replacement probability $w_{i j}$ that the offspring of the individual in $i$ replaces that in $j$. However, an important constraint in the "Birth-death" model of Ref. [4] (also known as biased invasion process [11]) is $\sum_{j} w_{i j}=1$ for all $i$, because replacement includes birth, migration, and death at once, and population size is constant. By contrast, migration rates $m_{i j}$ in our model are all independent.
A generalized circulation theorem holds for our model [20], in the spirit of Ref. [4]. Specifically, a population of $D$ demes on a graph has the same mutant fixation probability as the clique if and only if, for all nodes of the graph, the total outgoing migration rate is equal to the total incoming migration rate.

Thus, we expect fixation probabilities in our model to map to those of Ref. [4] for circulations or if $\sum_{j} m_{i j}$ is independent of $i$, but to potentially differ otherwise. We now consider specific graphs with strong symmetries.

Clique and cycle.-In the clique (or island model $[5,6]$ ), all demes are equivalent and connected to all others with identical migration rates $m$ per individual (Fig. 1, upper inset). Starting from one fully mutant deme and $D-1$ fully wild-type demes, the fixation probability $\Phi_{1}^{\text {clique }}$ of the mutant reads [20] (proof inspired by Refs. [9,30])

$$
\Phi_{1}^{\text {clique }}=\frac{1-\gamma}{1-\gamma^{D}}
$$

with

$$
\gamma=\frac{N_{W} \rho_{W}}{N_{M} \rho_{M}}
$$

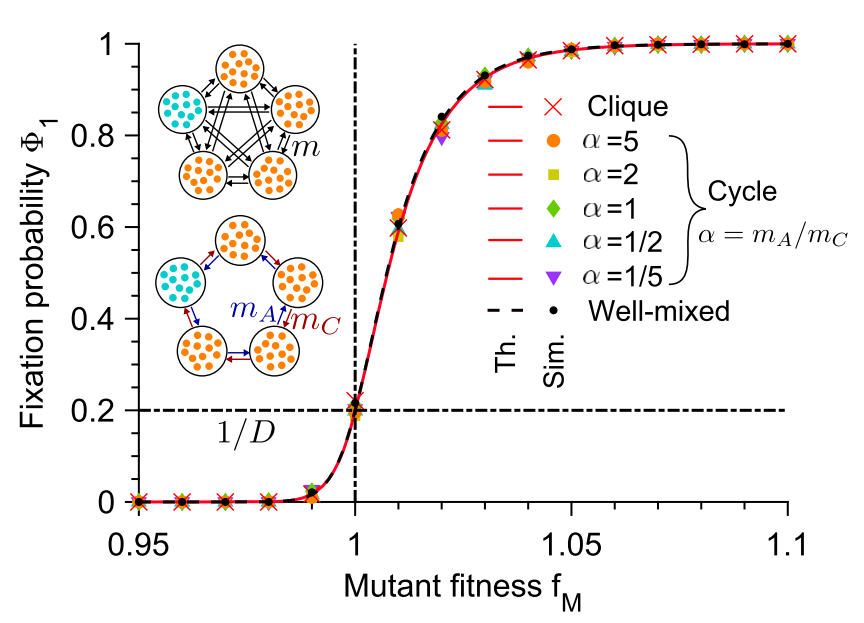

FIG. 1. Fixation probability $\Phi_{1}$ of the mutant type versus mutant fitness $f_{M}$, for the clique (see upper inset), and for the cycle (see lower inset) with different migration rate asymmetries $\alpha=m_{A} / m_{C}$, starting with one fully mutant deme. Data for the well-mixed population is shown as reference, with same total population size and initial number of mutants. Markers are computed over $10^{3}$ stochastic simulation realizations. Curves represent analytical predictions, Eq. (2) for the cycle and the clique, and Eq. (S15) in the Supplemental Material [20] for the well-mixed population [31,32]. Vertical dash-dotted lines indicate the neutral case $f_{M}=f_{W}$, and horizontal dash-dotted lines represent the neutral fixation probability. Parameter values are $D=5, K=100, f_{W}=1, g_{W}=g_{M}=0.1$ in both panels. In simulations, for the clique, $m=10^{-6}$; for the cycle, from top to bottom, $\left(m_{A}, m_{C}\right) \times 10^{6}=(1,5),(1,2),(1,1),(2,1),(5,1)$. 
where $N_{W}$ (respectively $N_{M}$ ) is the deterministic steadystate size of a wild-type (respectively mutant) deme and $\rho_{W}$ (respectively $\rho_{M}$ ) is the fixation probability of a wildtype (respectively mutant) microbe in a mutant (respectively wild-type) deme. This result is independent of migration rate $m$, and Eq. (2) has the exact same form as the fixation probability of a single mutant in a wellmixed population of fixed size $D$ in the Moran model [31,32], but with $\gamma$ playing the role of the ratio $f_{W} / f_{M}$, consistently with the formal mapping [Eq. (1)] between our model and that of Ref. [4] where $N \rho$ plays the part of fitness. $\Phi_{1}^{\text {clique }}$ is plotted versus $f_{M}$ in Fig. 1, showing excellent agreement between Eq. (2) and our stochastic simulation results. Moreover, this fixation probability is very close to that in a well-mixed population. We show [20] that the clique is a slight suppressor of selection, but that modeling migrations as exchanges of individuals and assuming $N_{M}=N_{W}$ exactly recovers the well-mixed result, consistent with results on symmetric migrations $[7,8]$.

Another graph where all demes are equivalent is the cycle. Clockwise and anticlockwise migrations can have different rates, denoted respectively by $m_{C}$ and $m_{A}$ (Fig. 1, lower inset). The cycle resembles the circular steppingstone model [7], but can feature asymmetric migrations. We show [20] that the fixation probability $\Phi_{1}^{\text {cycle }}$ is the same as for the clique [Eq. (2)], as corroborated by our simulations (see Fig. 1). Indeed, the cycle is a circulation. In particular, migration rates do not impact $\Phi_{1}^{\text {cycle }}$.

Star.-In the star, a central node is connected to all others, called leaves. An individual can migrate from a leaf to the center with migration rate $m_{I}$ and vice versa with rate $m_{O}$ (Fig. 2, inset). The mutant fixation probability can be expressed exactly as a function of $D, \alpha=m_{I} / m_{O}$ and $\gamma$ defined in Eq. (3) (proof in the Supplemental Material [20] inspired by Ref. [33]):

$$
\Phi_{1}^{\mathrm{star}}=\frac{\left(1-\gamma^{2}\right)\left[\gamma+\alpha D+\gamma \alpha^{2}(D-1)\right]}{D(\alpha+\gamma)\left[1+\alpha \gamma-\gamma^{D}(\alpha+\gamma)^{2-D}(1+\alpha \gamma)^{D-1}\right]} .
$$

Figure 2(a) shows the fixation probability $\Phi_{1}^{\text {star }}$ of the mutant type for different values of migration asymmetry $\alpha=m_{I} / m_{O}$, with very good agreement between Eq. (4) and our simulations. If $\alpha<1$, the star suppresses selection compared with the clique, while for $\alpha>1$ it slightly amplifies selection in some range of mutant fitness $f_{M}$ [20]. For $\alpha=1, \Phi_{1}^{\text {star }}$ reduces to the fixation probability of the clique [Eq. (2)] [20]. Consistently, the star is a circulation for $\alpha=1$. Stronger amplification for $\alpha>1$ is obtained for large $D$ [Fig. 2(b)]. Qualitatively, for large $D$, mutants very likely start in a leaf. If $\alpha$ is large, they often spread to the center, which helps the fit mutants take over. Conversely, if $\alpha$ is small, the center often invades the leaves, thus preventing any mutant originating in a leaf from fixing. Results with mutants starting in a specific deme are also shown in Ref. [20].

Imposing that $\sum_{j} m_{i j}$ is independent of $i$ amounts to imposing $\alpha=D-1$ in the star [20]. Then, Eq. (4) reduces to the formula [33] obtained in the model of Ref. [4], with $\gamma$ in Eq. (3) playing the role of $f_{W} / f_{M}$ [20], as per our general mapping [Eq. (1)]. The celebrated amplification property of the star in the large $D$ limit $[4,34]$ is thus exactly recovered in our model for $\alpha=D-1$.

While the star is an amplifier for large $D$ in the model of Ref. [4], it can either suppress or an amplify selection,
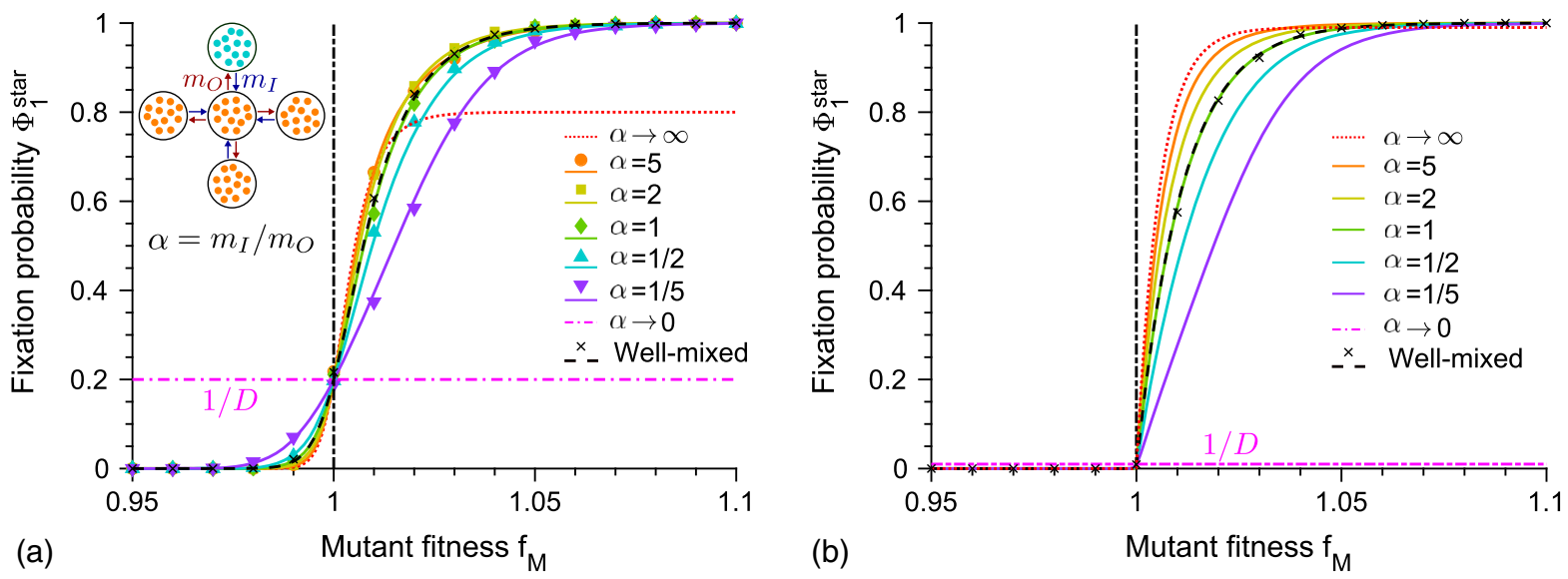

FIG. 2. Fixation probability $\Phi_{1}^{\text {star }}$ of the mutant type in a star graph versus mutant fitness $f_{M}$, starting with one fully mutant deme chosen uniformly at random, for different migration rate asymmetries $\alpha=m_{I} / m_{O}$. Number of demes: $D=5$ (a) and $D=100$ (b). Data for the well-mixed population is shown as reference, with same total population size and initial number of mutants. Markers are computed over $2 \times 10^{3}$ stochastic simulation realizations. Curves represent analytical predictions in Eq. (4). Vertical dash-dotted lines indicate the neutral case $f_{W}=f_{M}$. Parameter values are $K=100, f_{W}=1, g_{W}=g_{M}=0.1$. Panel (a): from top to bottom, $\left(m_{I}, m_{O}\right) \times 10^{6}=(5,1),(2,1),(1,1),(1,2),(1,5)$ in simulations. 


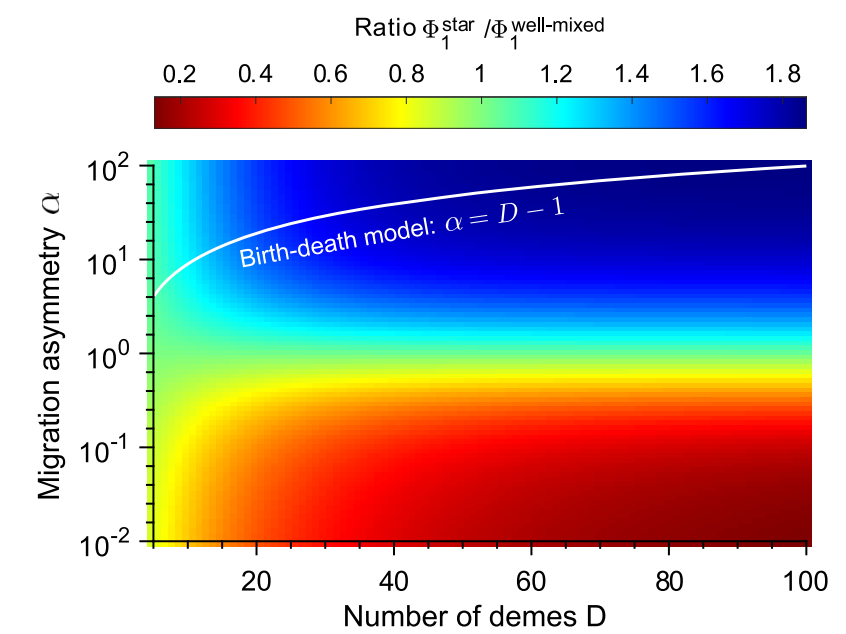

FIG. 3. Amplification and suppression properties for the star. Heat map of the ratio of the fixation probability $\Phi_{1}^{\text {star }}$ of the mutant type in a star graph to that $\Phi_{1}^{\text {well-mixed }}$ in a well-mixed population with same total population size and initial number of mutants, versus number $D$ of demes and migration rate asymmetry $\alpha=m_{I} / m_{O}$. The star is initialized with one fully mutant deme chosen uniformly at random. Data are from the analytical formula in Eq. (4) for the star, and in Eq. (S15) in the Supplemental Material [20] for the well-mixed population. Parameter values are $K=100, f_{W}=1, f_{M}=1.001, g_{W}=g_{M}=0.1$.

depending on $\alpha$, in our model where $D$ and $\alpha$ are two independent parameters. Figure 3 shows that restricting to $\alpha=D-1$ yields amplification. In models with one individual per node, the star is an amplifier for large $D$ for the Birth-death dynamics ("update rule"), where one individual is chosen to divide, and its offspring replaces one of its neighbors [4], but a suppressor for the death-Birth dynamics (or biased voter model [11]), where one individual is chosen to die and is replaced by the offspring of one of its neighbors (selection being on division rates in both cases, as denoted by the uppercase "Birth" [13], and resulting in global selection in the Birth-death case and local selection in the death-Birth case) $[13,29,35,36]$. Consistently, the latter dynamics would yield $\alpha=1 /(D-1)$.

Comparison to Ref. [16].—A model generalizing Ref. [4] to graphs where each node contains a deme with fixed population size was introduced in Ref. [16] (see also Refs. [15,18,37]). In this model, as in Ref. [4], each elementary event comprises a birth in one deme and a death in another one, yielding Birth-death and death-Birth models that give different results. Rare migrations in our model correspond to strong self-loops (migrations to the original deme) in the model of Ref. [16]. For the star [28], we show [20] that by matching migration-to-division rate ratios in each deme, both models yield similar simulation results. However, even then, a difference is that the death rate (respectively birth rate) is not homogeneous across demes in the Birth-death (respectively death-Birth) models of Ref. [16], unless migrations are symmetric. Our model allows more realistic choices.
Discussion.-We developed a model of spatially structured microbial populations on graphs where migration, birth, and death are independent events. We showed that for rare migrations, the star graph continuously transitions between amplifying and suppressing natural selection as migration rate asymmetry is varied. This elucidates the apparent paradox in existing models, where the star, like many random graphs [13], is an amplifier in the Birth-death dynamics and a suppressor in the death-Birth dynamics $[13,29,35,36]$. We found a mapping between our model and that of Ref. [4], under a constraint on migration rates. Models with one individual per node require making specific choices on the microscopic dynamics ("update rule"), which constrain migration rates. By lifting this constraint, our model reconciles and generalizes previous results, showing that migration rate asymmetry is key to whether a given population structure amplifies or suppresses natural selection. This crucial role of migration asymmetry is consistent with the fact that structures with symmetric migrations do not affect fixation probabilities [7,8].

Birth-death dynamics may be realistic for extreme resource limitation, such that one birth causes one death, while death-Birth dynamics may better model cases where death frees resources, e.g., light for plants [35,38]. However, in general, in a microbial population, population size is not strictly fixed, and the order of birth and death events is not set. In our more universal model, the results do not hinge on a modeling choice made for microscopic dynamics. Instead, they depend on a quantity that can be directly set or measured in experiments, namely migration rate asymmetry. The differences between Birth-death and death-Birth dynamics are major for mutant fixation probabilities, but also in evolutionary game theory, where spatial structure can promote the evolution of cooperation in the latter case, but not in the former [39-41]. Previous efforts were made to generalize beyond these dynamics by allowing both types of update to occur in given proportions $[42,43]$. Interestingly, it was recently shown that no general amplification of selection can occur when even a small proportion of death-Birth events occurs [43], in contrast with the Birth-death case. Conversely, in our model, the amplification property of the star graph in the large-size limit is preserved, but for sufficient migration asymmetry.

While our focus was on mutant fixation probabilities, our model can be employed to investigate fixation times and evolution rate $[18,44-50]$. It can also address more complex population structures [29,51], e.g., motivated by within-host or between-host pathogen dynamics [52]. Our study can be extended beyond the regime of rare migrations [53], and to models of evolutionary game theory, as well as to diploid organisms $[10,26,27,54,55]$. Finally, our work allows direct comparisons with quantitative experiments [19]. Other experiments could be performed using, e.g., microfluidic devices allowing one to control the flow between different populations [56], or microtiter plates 
where dilutions and migrations can be performed via a liquid-handling robot [57-59]. Applications in biotechnology could be envisioned, e.g., amplifying in vivo selection in the directed evolution of biomolecules [60].

This project has received funding from the European Research Council (ERC) under the European Union's Horizon 2020 research and innovation program (Grant Agreement No. 851173, to A.-F. B.). L. M. acknowledges funding by a graduate fellowship from École Doctorale Physique en Île-de-France. L. M. thanks his grandfather, Jean Polard, for inspiration.

* Corresponding author. anne-florence.bitbol@epfl.ch

${ }^{\dagger}$ Present address: Institute of Ecology and Evolution, University of Bern, Baltzerstrasse 6, CH-3012 Bern, Switzerland.

*Present address: Institute of Bioengineering, School of Life Sciences, École Polytechnique Fédérale de Lausanne (EPFL), CH-1015 Lausanne, Switzerland.

[1] G. van Marle, M. J. Gill, D. Kolodka, L. McManus, T. Grant, and D. L. Church, Retrovirology 4, 87 (2007).

[2] G. Schnell, R. W. Price, S. R., and S. Spudich, J. Virol. 84, 2395 (2010).

[3] F. Herrerías-Azcué, V. Pérez-Muñuzuri, and T. Galla, Sci. Rep. 8, 4068 (2018).

[4] E. Lieberman, C. Hauert, and M. A. Nowak, Nature (London) 433, 312 (2005).

[5] S. Wright, Genetics 16, 97 (1931).

[6] M. Kimura and G. H. Weiss, Genetics 49, 561 (1964).

[7] T. Maruyama, Genet. Res. 15, 221 (1970).

[8] T. Maruyama, Theor. Popul. Biol. 5, 148 (1974).

[9] M. Slatkin, Evolution 35, 477 (1981).

[10] N. Barton, Genet. Res. 62, 149 (1993).

[11] T. Antal, S. Redner, and V. Sood, Phys. Rev. Lett. 96, 188104 (2006).

[12] K. Kaveh, N. L. Komarova, and M. Kohandel, R. Soc. Open Sci. 2, 140465 (2015).

[13] L. Hindersin and A. Traulsen, PLoS Comput. Biol. 11, e1004437 (2015).

[14] K. Pattni, M. Broom, J. Rychtár̆, and L. J. Silvers, Proc. R. Soc. A 471, 20150334 (2015).

[15] P. R. A. Campos, J. Combadão, F. Dionisio, and I. Gordo, Phys. Rev. E 74, 042901 (2006).

[16] B. Houchmandzadeh and M. Vallade, New J. Phys. 13, 073020 (2011).

[17] B. Houchmandzadeh and M. Vallade, BioSystems 112, 49 (2013).

[18] G. W. Constable and A. J. McKane, J. Theor. Biol. 358, 149 (2014).

[19] P. Chakraborty, L. R. Nemzer, and R. Kassen, BioRxiv https://doi.org/10.1101/2021.07.13.452242 (2021).

[20] See Supplemental Material at http://link.aps.org/ supplemental/10.1103/PhysRevLett.127.218102 for details, which includes Refs. [21-25].

[21] M. Vogels, R. Zoeckler, D. M. Stasiw, and L. C. Cerny, J. Biol. Phys. 3, 183 (1975).
[22] O. Ovaskainen and B. Meerson, Trends Ecol. Evol. 25, 643 (2010).

[23] T. L. Parsons and C. Quince, Theor. Popul. Biol. 72, 121 (2007).

[24] D. T. Gillespie, J. Comput. Phys. 22, 403 (1976).

[25] D. T. Gillespie, J. Phys. Chem. 81, 2340 (1977).

[26] M. C. Whitlock and N. H. Barton, Genetics 146, 427 (1997).

[27] M. C. Whitlock, Genetics 164, 767 (2003).

[28] B. Adlam, K. Chatterjee, and M. A. Nowak, Proc. R. Soc. A 471, 20150114 (2015).

[29] B. Allen, C. Sample, R. Jencks, J. Withers, P. Steinhagen, L. Brizuela, J. Kolodny, D. Parke, G. Lippner, and Y. A. Dementieva, PLoS Comput. Biol. 16, e1007529 (2020).

[30] A. Traulsen and C. Hauert, Stochastic evolutionary game dynamics, in Reviews of Nonlinear Dynamics and Complexity (John Wiley and Sons, Ltd, New York, 2010), Chap. 2, pp. 25-61.

[31] P. A. P. Moran, Math. Proc. Cambridge Philos. Soc. 54, 6071 (1958).

[32] W. J. Ewens, Mathematical Population Genetics (SpringerVerlag, Berlin, 1979).

[33] B. Broom and J. Rychtár, Proc. R. Soc. A 464, 2609 (2008).

[34] F. A. C. C. Chalub, J. Dyn. Games 3, 217 (2016).

[35] M. Frean and G. Baxter, Death-birth ordering and suppression of fitness in networks (2008), https://homepages.ecs .vuw.ac.nz/ marcus/manuscripts/FreanBaxterJTB.pdf.

[36] C. Hadjichrysanthou, M. Broom, and J. Rychtář, Dyn. Games Appl. 1, 386 (2011).

[37] A. Traulsen, A. M. Sengupta, and M. A. Nowak, J. Theor. Biol. 235, 393 (2005).

[38] D. Alonso, R. S. Etienne, and A. J. McKane, Trends Ecol. Evol. 21, 451 (2006).

[39] H. Ohtsuki, C. Hauert, E. Lieberman, and M. A. Nowak, Nature (London) 441, 502 (2006).

[40] P. D. Taylor, T. Day, and G. Wild, Nature (London) 447, 469 (2007).

[41] F. Débarre, J. Theor. Biol. 420, 26 (2017).

[42] J. Zukewich, V. Kurella, M. Doebeli, and C. Hauert, PLoS One 8, e54639 (2013).

[43] J. Tkadlec, A. Pavlogiannis, K. Chatterjee, and M. A. Nowak, PLoS Comput. Biol. 16, e1007494 (2020).

[44] G. J. Baxter, R. A. Blythe, and A. J. McKane, Phys. Rev. Lett. 101, 258701 (2008).

[45] M. Frean, P. B. Rainey, and A. Traulsen, Proc. Biol. Sci. 280, 20130211 (2013).

[46] C. Hauert, Y.-T. Chen, and L. A. Imhof, J. Stat. Phys. 156, 739 (2014).

[47] L. Hindersin and A. Traulsen, J. R. Soc. Interface 11, 20140606 (2014).

[48] P. Lombardo, A. Gambassi, and L. Dall'Asta, Phys. Rev. Lett. 112, 148101 (2014).

[49] B. Allen, C. Sample, Y. Dementieva, R. C. Medeiros, C. Paoletti, and M. A. Nowak, PLoS Comput. Biol. 11, e1004108 (2015).

[50] D. Hathcock and S. H. Strogatz, Phys. Rev. E 100, 012408 (2019).

[51] A. Pavlogiannis, J. Tkadlec, K. Chatterjee, and M. A. Nowak, Commun. Biol. 1, 71 (2018).

[52] F. Bansept, L. Marrec, A. F. Bitbol, and C. Loverdo, Evolution 73, 1077 (2019). 
[53] S. Yagoobi and A. Traulsen, Sci. Rep. 11, 17979 (2021).

[54] T. Nagylaki, J. Math. Biol. 9, 101 (1980).

[55] H. R. Pulliam, Am. Nat. 132, 652 (1988).

[56] K. W. Oh and C. H. Ahn, J. Micromech. Microeng. 16, R13 (2006).

[57] S. Kryazhimskiy, D. P. Rice, and M. M. Desai, Evolution 66, 1931 (2012).
[58] J. R. Nahum, P. Godfrey-Smith, B. N. Harding, J. H. Marcus, J. Carlson-Stevermer, and B. Kerr, Proc. Natl. Acad. Sci. U.S.A. 112, 7530 (2015).

[59] M. T. France and L. J. Forney, Am. Nat. 193, 503 (2019).

[60] F. H. Arnold and A. A. Volkov, Curr. Opin. Chem. Biol. 3, 54 (1999). 